\title{
Application of lamina replantation with ARCH plate fixation in thoracic and lumbar intraspinal tumors
}

\author{
DONG ZHOU*, LU-MING NONG* , GONG-MIN GAO, YU-QIN JIANG and NAN-WEI XU \\ Department of Orthopedics, The Second People's Hospital of Changzhou, Nanjing Medical University, \\ Changzhou, Jiangsu 213000, P.R. China
}

Received March 8, 2013; Accepted May 31, 2013

DOI: $10.3892 /$ etm.2013.1170

\begin{abstract}
The aim of the present study was to investigate the clinical effects of lamina replantation with ARCH plate fixation on patients with thoracic and lumbar intraspinal tumors, following laminectomy. Thirteen patients with thoracic and lumbar intraspinal tumors underwent total lamina replantation with ARCH plate fixation and repair of the supraspinous ligaments, following laminectomy and tumor enucleation. To investigate the clinical effect of lamina replantation with ARCH plate fixation, pre- and postoperative visual analog scale (VAS), and Oswestry Disability Index (ODI) scores were determined, and pre- and postoperative X-ray and magnetic resonance imaging (MRI) examinations were conducted. Computed tomography (CT) examinations were also included in the follow-up. No complications were observed pre- or postoperatively. The VAS and ODI results 2 weeks following surgery and at the final follow-up examination demonstrated a significant improvement compared with the corresponding preoperative results. The X-ray examination results indicated a satisfactory internal fixation location, without any characteristics of a fracture, lumbar scoliosis, kyphosis or instability. Following the surgery, the CT and MRI examination results demonstrated that healing of the lamina bone and repair of the supraspinous ligament had occurred without tumor recurrence or spinal epidural scar recompression. Two of the 13 cases were lost to follow-up. The results indicated that in patients with thoracic and lumbar intraspinal tumors, lamina replantation with ARCH plate fixation following total laminectomy is effective and provides thoracolumbar stability. Furthermore,
\end{abstract}

Correspondence to: Dr Lu-Ming Nong or Dr Nan-Wei Xu, Department of Orthopedics, The Second People's Hospital of Changzhou, Nanjing Medical University, 29 Xinglong Road, Changzhou, Jiangsu 213000, P.R. China

E-mail: lumingnong@163.com

E-mail: nanweixu@126.com

*Joint senior authorship

Key words: laminoplasty, intradural tumor, internal fixation this has been identified to be an effective technique for preventing intraspinal scar proliferation.

\section{Introduction}

The two basic principles in spine and spinal cord surgery are the complete resection of the intraspinal tumor and the restoration of spinal stability $(1,2)$. Multisegment laminectomy is typically performed to achieve complete exposure and resection of the intradural tumor. However, intraspinal epidural scar adhesions, spinal instability, kyphosis and additional complications are usually identified postoperatively $(3,4)$. Following the introduction of lamina replantation by Raimondi in 1976 (5), various technologies that assist in the procedure have been developed (6-9). The clinical effect of lamina replantation is limited due to a lack of appropriate internal fixation techniques. Although lamina replantation with fixation by a mini-plate is more commonly utilized in cervical treatment, this approach has also been used in thoracolumbar spinal surgery (10). However, further clinical cases and studies of the long-term effects of the method are required. The present study aimed to investigate the clinical effect of lamina replantation with ARCH plate fixation on patients diagnosed with thoracic and lumbar intraspinal tumors, following laminectomy.

\section{Materials and methods}

Patient data. From February 2009 to June 2010, 13 patients (32 segments) with thoracic and lumbar intraspinal tumors underwent lamina replantation with fixation using an $\mathrm{ARCH}$ plate (Synthes Inc., Wilmington, DE, USA) following laminectomy (Table I). Following the surgery, the majority of the patients experienced pain in the thoracic and lumbar regions during rest or when sleeping, along with lower limb numbness and weakness. In addition, the patients endured difficulties associated with urination or defecation. This study was conducted in accordance with the Declaration of Helsinki, and with approval from the ethics committee of The Second People's Hospital of Changzhou, Nanjing Medical University (Changzhou, China). Written informed consent was obtained from all participants.

Surgical technique. The patients were administered general anesthesia through tracheal intubation, and the surgery was 
Table I. Clinical data of patients.

\begin{tabular}{clclll}
\hline Case no. & Gender & Age (years) & Diagnosis & \multicolumn{1}{c}{ Position } & Replantation segments \\
\hline 1 & Male & 45 & Neurilemmoma & Thoracolumbar segment & 2 \\
2 & Female & 40 & Meningioma & Thoracic vertebra & 2 \\
3 & Female & 33 & Hemangioma & Thoracic vertebra & 3 \\
4 & Female & 46 & Neurilemmoma & Thoracolumbar segment & 3 \\
5 & Female & 28 & Astrocytoma & Thoracolumbar segment & 3 \\
6 & Male & 42 & Ependymoma & Thoracolumbar vertebra & 2 \\
7 & Female & 48 & Meningioma & Thoracic vertebra & 3 \\
8 & Male & 38 & Neurilemmoma & Thoracolumbar segment & 3 \\
9 & Male & 52 & Meningioma & Lumbar vertebrae & 2 \\
10 & Male & 46 & Meningioma & Thoracolumbar segment & 3 \\
11 & Female & 33 & Meningioma & Thoracolumbar segment & \\
12 & Female & 36 & Ependymoma & Lumbar vertebrae & \\
\hline
\end{tabular}

performed with patients in the prone position. A medial longitudinal incision was made along the thoracic and lumbar spine, and the bilateral sacrospinalis were separated to the small joints. Based on the location of the tumor, which was determined by MRI, the spinous process and vertebral lamina that required cutting were exposed, while the supra- and interspinous ligaments remained intact. The cortex of the lamina was cut $(2-3 \mathrm{~mm})$ with a bone drill between the lateral lamina and the inner small joints, and an osteotome was then used to cut through the lamina. The supra- and interspinous ligaments, as well as the ligamentum flavum of the tail were cut in order to isolate the spinous ligament complex. The spinous ligament complex was then turned over and fixed in the head end. The tumor was exposed and removed, which resulted in a reduction in spinal cord compression. The spinous ligament complex was reset, and the inter- and supraspinous ligaments were sutured with polydioxane monofilament synthetic (PDS) II absorbable sutures (DePuy Orthopaedics, Inc., Warsaw, IN, USA). An ARCH steel plate of the appropriate size and shape was inserted and fixed bilaterally with titanium screws, following the resetting of the lamina. The tube placement was drained, and then the incision was washed and closed at each layer.

Antibiotics were routinely administered intra- and postoperatively. On the first day following surgery, the patients were required to perform back muscle exercises in bed. A girdle brace was introduced to patients that performed exercise in bed for three days following the surgery. Two weeks following surgery, the patients were permitted out of bed with the assistance of a girdle brace. The waist brace was removed one month following the surgery.

Therapeutic evaluations. The visual analog scale (VAS) and the Oswestry Disability Index (ODI) were used for pre- and postoperative therapeutic evaluation.

Imaging evaluation. To determine the level of internal fixation and spinal stability, lumbar spine X-rays were performed in the posterior-anterior, lateral and flexion-extension positions, one day prior to the surgery, and at 2 weeks, and 1, 3, 6 and 12 months following the surgery. A computed tomography (CT) scan was performed three months following surgery and monthly thereafter, until the lamina bones had fused. Furthermore, the bone growth of the regrafted lamina was evaluated. Six months following the surgery, magnetic resonance imaging (MRI) was performed to detect tumor recurrence and scar oppression in the spinal canal, and the repair of the ligaments.

Statistical analysis. The VAS and ODI scores were expressed as the mean \pm standard deviation. A paired t-test was performed using the SPSS software, version 11.0 (SPSS, Inc., Chicago, IL, USA). $\mathrm{P}<0.05$ and $\mathrm{P}<0.01$ were considered to indicate a statistically significant difference.

\section{Results}

Clinical effects. The thirteen patients in this study underwent successful surgery and demonstrated primary healing. One patient with ependymoma who developed postoperative cerebral spinal fluid (CSF) leakage spontaneously recovered following conservative treatment. The development of complications following the surgery did not occur in the other patients. In addition, the thoracolumbar and back pain was significantly relieved or disappeared following the surgery, while the numbness of the limbs also decreased in intensity.

One month following the surgery, two of the cases were lost to follow-up. The follow-up period in the remaining 11 patients was 9-22 months, with a final follow-up period of $>3$ months. The VAS and ODI scores of the patients are shown in Table II.

Biomechanical test results. In the present study, a biomechanical test of the lumbar lamina replantation with ARCH steel plate fixation was completed, which showed that following the surgery the vertebral strain increased by $36-60 \%$, the transverse transposition increased by $38-49 \%$, 
Table II. VAS and ODI scores.

\begin{tabular}{lcrr}
\hline Scale & One day prior to surgery & Two weeks following surgery & Final follow-up \\
\hline VAS & $8.8 \pm 1.5$ & $2.8 \pm 1.3^{\mathrm{a}}$ & $1.1 \pm 0.4^{\mathrm{a}, \mathrm{b}}$ \\
ODI $(\%)$ & $89.3 \pm 9.2$ & $52.8 \pm 6.5^{\mathrm{c}}$ & $10.8 \pm 2.3^{\mathrm{a}, \mathrm{d}}$ \\
\hline
\end{tabular}

Scores are mean \pm standard deviation. ${ }^{a} \mathrm{P}<0.01$ vs. preoperative score; ${ }^{\mathrm{b}} \mathrm{P}<0.05$ vs. score 2 weeks following surgery; ${ }^{\mathrm{c}} \mathrm{P}<0.05$ vs. preoperative score; ${ }^{\mathrm{P}}<0.01$ vs. score 2 weeks following surgery. VAS, visual analog scale; ODI, Oswestry Disability Index.

A

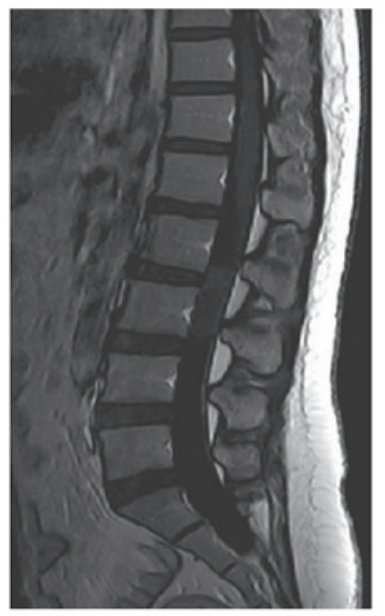

D

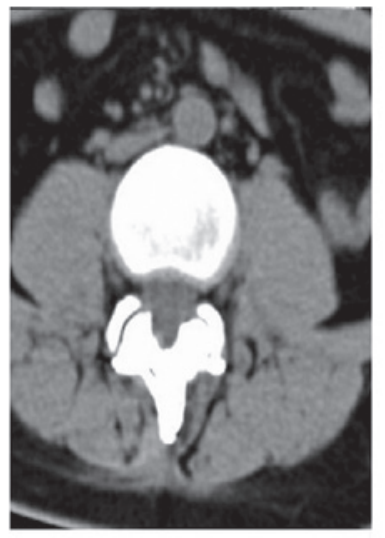

B

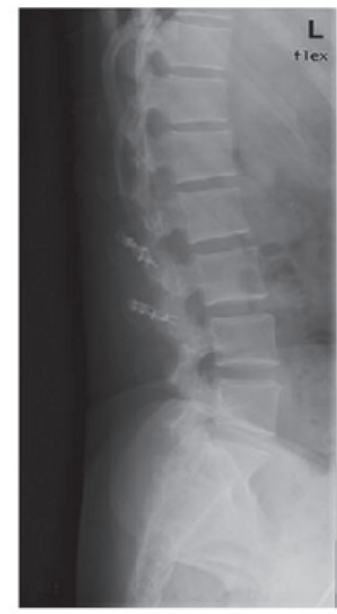

$\mathbf{E}$

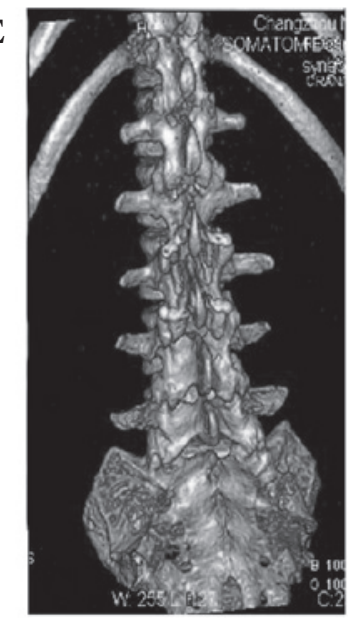

C

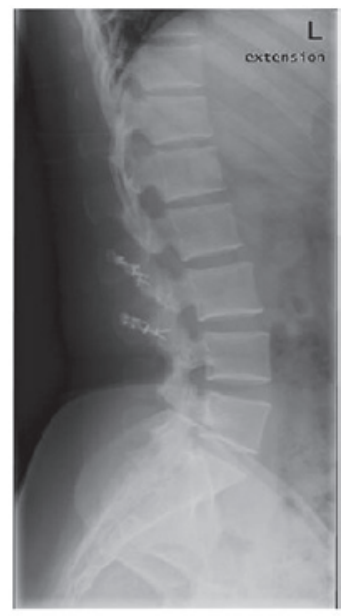

$\mathbf{F}$

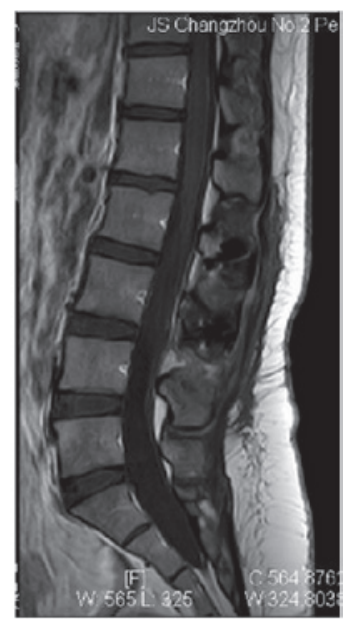

Figure 1. Case 2. Ependymoma at the L2-3 level. (A) Prior to surgery, magnetic resonance imaging (MRI) identified that the L2-3 spinal space was occupied by the tumor. (B and C) One year following the surgery, X-ray examination indicated no fixation transposition or fracture, lumbar instability or kyphosis. Three months following the surgery, favorable lamina bone fusion was indicated by (D) a postoperative computed tomography (CT) scan and (E) 3-D reconstruction. (F) Six months following the surgery, no intraspinal scar adhesions or restenosis were identified by MRI.

the stiffness decreased by $26-57 \%$ and the carrying capacity decreased by $32-45 \%$. In addition, lamina replantation with plate fixation resulted in similar levels of vertebral strain, transposition, stiffness and carrying capacity, following surgery, compared with normal levels (difference, 4-11\%; $\mathrm{P}<0.05)$.

MRI findings. Following the surgery, no fixation transposition or fracture, lumbar instability or kyphosis was identified. In patients with a follow-up period of $>6$ months, favorable healing of the vertebral lamina without bone restenosis or intraspinal epidural scar adhesions was observed (Fig. 1).

\section{Discussion}

It has been indicated that a posterior approach to the spine may provide access to a wider area of the spine during surgery, and therefore reveal the intraspinal tumor for complete resection. However, the clinical application of a posterior approach is limited due to the wide removal of bone and ligaments, as well as the high risk of peridural adhesions and spinal cord injury, which are associated with weak bone protection $(11,12)$.

Previous short-term follow-up studies on the laminectomy procedure demonstrated a high degree of satisfaction and spinal stability $(13,14)$. However, in long-term studies, the satis- 
faction rate decreased to $<60 \%$, as lumbar instability resulted in chronic lower back pain $(15,16)$. A long-term study by Mullin et al (17) identified that $54 \%$ of patients with dynamic lumbar instability had previously undergone total laminectomy. In addition, Papagelopoulos et al (14) revealed that $28 \%$ of young patients ( $\leq 30$ years) treated with thoracolumbar multisegment laminectomy exhibited spinal deformity. Iida et al (11) demonstrated that extensive laminectomy induced intervertebral instability. Moreover, a posterior structure resection was more likely than nucleus pulposus removal to cause postoperative spinal instability. However, Tsuji et al (18) demonstrated that lower back pain and sensory disturbance were marginally improved following laminectomy.

Laminoplasty is considered to be important for young patients with benign tumors, so as to avoid postoperative complications associated with laminectomy, such as refractory back pain and spinal deformity $(5,19)$. Two-stage anteroposterior spinal fusion and internal fixation may provide favorable clinical outcomes compared with laminectomy; single-stage extensive laminectomy may cause posterior bone deficiency (12). In China, single-stage laminectomy, nail-stick system fixation and bone fusion surgery have been used to achieve immediate spinal stability. These procedures have resulted in a large surgical field, segment movement disorder and peripheral segment degeneration (20).

The long-term postoperative complications have not been increased; however, numerous scholars have adopted various techniques in order to treat thoracic and lumbar intraspinal tumors $(2,6-9,13,21,22)$. Menku et al proposed the application of a mini-plate in thoracic and lumbar laminoplasty, as opposed to its traditional usage in the cervix, to fix the replanted lamina, and to provide immediate stability and a smaller surgical incision (10).

Results of biomechanical tests showed that the technique of lamina replantation with $\mathrm{ARCH}$ plate fixation was able to improve spinal stability, compressive resistance and anti-bending, -shearing and -rotation abilities.

Numerous studies that aimed to prevent postoperative epidural adhesion, such as by the use of adipose tissue, amniotic membranes, silicon films and silicon rubber sheets, and hormone and anti-inflammatory drug instillation, have not been successful $(11,12,24-26)$. However, we propose that the vertebral plate is an effective and safe isolation method that prevents scar adhesion in lamina replantation fixation.

A previous study revealed that supra- and interspinal ligaments were well innervated, and that this innervation may form the basis of neurological feedback mechanisms for the protection and stability of the spine (27). Studies by Hotta (28) and Newman (29) demonstrated the importance of the supraand interspinal ligaments in the enhancement of spinal flexion stability. In addition, Sano et al (30) and Joson et al (31) proposed methods of conserving the supraspinous ligament during laminectomy. Hirofuji et al (32) suggested reconstruction of the supra- and interspinous ligaments using artificial ligaments. In the present study, unilateral supra- and interspinous ligaments were cut, reversely rotated with the spinous process and the lamina, and repaired with PDS II absorbable sutures following tumor removal and lamina replantation.

Lamina replantation with $\mathrm{ARCH}$ plate fixation and ligament repair were adopted in the current study, as the technique allows for smaller surgical incisions, allows bone protecting the spinal cord to be conserved, prevents spinal instability and kyphosis, and preserves the spinous process during lamina replantation, resulting in a favorable appearance. In addition, the restoration of the ligament-nerve-muscle reflex system of the supra- and interspinous ligaments aids lower back movement. Furthermore, the technique prevents epidural adhesion following laminectomy. Moreover, a second surgical procedure is then safer and simpler, as the posterior bony structure is preserved. Lamina replantation enables muscle and soft tissue attachment, increasing postoperative perispinal muscle function. Additionally, the biomechanical environment of the surgical segments is recovered, which prevents movement loss and peripheral segment degeneration.

Thoracolumbar laminoplasty using the posterior approach is beneficial, as it retains the posterior spinal structures and prevents postoperative bleeding, scar adhesions, instability, subluxation and kyphosis. In addition, it provides uncomplicated access when further surgery is required. Lamina replantation with titanium plate fixation has been demonstrated to be a favorable surgical procedure that is not limited by the patient's age, the surgical site or the number of impaired segments.

\section{Acknowledgements}

This study was supported by the Changzhou Science and Technology Bureau Project (grant no. CJ20112017) and the key project of Changzhou city Health Bureau (grant no. ZD201103).

\section{References}

1. Love JG: Laminectomy for the removal of spinal cord tumors. J Neurosurg 25: 116-121, 1966.

2. Wiedemayer H, Sandalcioglu IE, Aalders M, Wiedemayer H, Floerke M and Stolke D: Reconstruction of the laminar roof with miniplates for a posterior approach in intraspinal surgery: technical considerations and critical evaluation of follow-up results. Spine (Phila Pa 1976) 29: E333-E342, 2004.

3. Jönsson B, Annertz M, Sjöberg C and Strömqvist B: A prospective and consecutive study of surgically treated lumbar spinal stenosis. Part II: Five years follow-up by an independent observer. Spine (Phila Pa 1976) 22: 2938-2944, 1997.

4. Kondo E and Yamada K: Osteoplastic laminectomy for lumbar disc protrusion. Arch Jap Chir 23: 287-294, 1954.

5. Raimondi AJ, Gutierrez FA and Di Rocco C: Laminotomy and total reconstruction of the posterior spinal arch for spinal canal surgery in childhood. J Neurosurg 45: 555-569, 1976.

6. Fidler MW and Bongartz EB: Laminar removal and replacement: a technique for the removal of epidural tumor. Spine (Phila Pa 1976) 13: 218-220, 1988.

7. Goel A and Deogaonkar M: Thoracic laminoplasty using spinous processes - technical note. Neurol Med Chir 36: 659-661, 1996

8. Matsui H, Kanamori M and Miaki K: Expansive laminoplasty for lumbar intradural lipoma. Int Orthop 21: 185-187, 1997.

9. Mimatsu K: New laminoplasty after thoracic and lumbar laminectomy. J Spinal Disord 10: 20-26, 1997.

10. Menku A, Koc RK, Oktem IS, Tucer B and Kurtsoy A: Laminoplasty with miniplates for posterior approach in thoracic and lumbar intraspinal surgery. Turk Neurosurg 20: 27-32, 2010.

11. Iida Y, Kataoka O, Sho T, Sumi M, Hirose T, Bessho Y and Kobayashi D: Postoperative lumbar spinal instability occurring or progressing secondary to laminectomy. Spine (Phila Pa 1976) 15: $1186-1189,1990$.

12. Lonstein JE: Postlaminectomy spinal deformity. In: Moe's Textbook of Scoliosis and Other Spinal Deformities. Lonstein JE, Bradford DS, Winter RB and Ogilvie JW (eds). 3rd edition. WB Saunders, Philadelphia, pp506-515, 1995. 
13. Airaksinen O, Herno A, Turunen V, Saari T and Suomlainen O: Surgical outcome of 438 patients treated surgically for lumbar spinal stenosis. Spine (Phila Pa 1976) 22: 2278-2282, 1997.

14. Papagelopoulos PJ, Peterson HA, Ebersold MJ, Emmanuel PR, Choudhury SN and Quast LM: Spinal column deformity and instability after lumbar or thoracolumbar laminectomy for intraspinal tumors in children and young adults. Spine (Phila Pa 1976) 22: 442-451, 1997.

15. Katz JN, Lipson SJ, Chang LC, Levine SA, Fossel AH and Liang MH: Seven- to 10-year outcome of decompressive surgery for degenerative lumbar spinal stenosis. Spine (Phila Pa 1976) 21: 92-98, 1996.

16. Scholz M, Firsching R and Lanksch WR: Long term follow-up in lumbar spinal stenosis. Spinal Cord 36: 200-204, 1998.

17. Mullin BB, Rea GL, Irsik R, Catton M and Miner ME: The effect of postlaminectomy spinal instability on the outcome of lumbar spinal stenosis patients. J Spinal Disord 9: 107-116, 1996.

18. Tsuji M, Kurihara A, Urtsuji Y, Shoda E and Mizuno T: The results of surgical treatment for degenerative spondylolisthetic stenosis. Clin Orthop Surg 25: 455-461, 1990.

19. Shikata J, Yamamuro T, Shimizu K and Saito T: Combined laminoplasty and posterolateral fusion for spinal canal surgery in children and adolescents. Clin Orthop Relat Res 259: 92-99, 1990.

20. Lin B, Huang ZZ, Guo ZM, Liu H and Sha M: Surgical treatment for multisegmental intraspinal tumors of the spine. J Clin Orthop 13: 365-368, 2010 (In Chinese).

21. Hara M, Takayasu M, Takagi T and Yoshida J: En bloc laminoplasty performed with threadwire saw. Neurosurgery 48 235-239, 2001.

22. Kawahara N, Tomita K, Shinya Y, et al: Recapping T-saw laminoplasty for spinal cord tumors. Spine (Phila Pa 1976) 24: $1363-1370,1999$.
23. Murakami H, Mamune N, Isaki H, Asazuma T and Yamagishi M: A case report of giant cauda equina tumor presented with minor symptoms. Sekituisekizui 11: 53-56, 1998.

24. La Rocca H and Macnab I: The laminectomy membrane. Studies in its evolution, characteristics, effects and prophylaxis in dogs. J Bone Joint Surg Br 56B: 545-550, 1974.

25. Winter RB and Hall JE: Kyphosis in childhood and adolescence. Spine (Phila Pa 1976) 3: 285-308, 1978

26. Yasuoka S, Peterson HA and MacCarty CS: Incidence of spinal column deformity after multilevel laminectomy in children and adults. J Neurosurg 57: 441-445, 1982.

27. Jiang H, Russell IG, Raso VJ, Moreau MJ, Hill DL and Bagnall KM: The nature and distribution of the innervation of human supraspinal and interspinal ligaments. Spine (Phila Pa 1976) 20: 869-876, 1995.

28. Hotta H: An experimental study on stability of human spine, especially the role of the lumbar ligaments. J Jpn OrthopAssoc 50: 1-14, 1976.

29. Newman PH: Sprung back. J Bone Joint Surg Br 34B: 30-37, 1952.

30. Sano S, Masuda A, Kabata K, Mitsui H and Kunoki J: Laminectomy with spinous process reattachment - preliminary report. Orthop Surg Traumatol 26: 1227-1230, 1983.

31. Joson RM and McCormik KJ: Preservation of the supraspinous ligament for spinal stenosis: a technical note. Neurosurgery 21 420-422, 1987.

32. Hirofuji E, Tanaka K and Nakano A: Ligamentous reconstruction with artificial ligament to prevent the unstable lumbar spine. Clin Orthop Surg 25: 501-506, 1990 . 